09

\title{
Использование методов голографической подповерхностной радиолокации для неразрушающего контроля диэлектрических конструкций
}

\author{
(C) С.И. Ивашов, А.С. Бугаев, А.В. Журавлев, В.В. Разевиг, М.А. Чиж, А.И. Ивашов
}

Московский государственный технический университет им. Н.Э. Баумана, 105005 Москва, Россия

e-mail: sivashov@rslab.ru

(Поступило в Редакцию 11 мая 2017 г.)

Описан метод голографической подповерхностной радиолокации и проведено его сравнение с традиционной технологией импульсных радиолокаторов. Приведены основные соотношения для восстановления комплексных СВЧ голограмм. Перечислены основные области применения данной технологии. На примере диагностики пенополиуретановых покрытий объектов ракетно-космической техники продемонстрирована эффективность использования голографических подповерхностных радиолокаторов. Выполнено сравнение результатов восстановления комплексных и амплитудных радиоголограмм. Показано, что качество изображений, полученных в результате восстановления комплексных радиоголограмм, выше по сравнению с амплитудными.

DOI: 10.21883/JTF.2018.02.45420.2330

\section{Введение}

Подповерхностная радиолокация в настоящее время стала общепризнанным методом обследования оптически непрозрачных сред $[1,2]$, который находит применение в самых различных областях. Перечислим некоторые из них: инженерная геология [3], обнаружение мин [4-6], обследование строительных конструкций и объектов культурного наследия [7], неразрушающий контроль диэлектрических материалов [8] и др.

Традиционно на практике используются подповерхностные локаторы импульсного типа, излучающие сигнал, близкий по форме к одному периоду синусоиды. Практически все серийно выпускаемые в настоящее время подповерхностные локаторы относятся к этому типу, что объясняется рядом причин. Так, основным преимуществом этого типа приборов является их способность зондировать среду с высоким удельным поглощением на относительно бо́льших глубинах по сравнению с локаторами, излучающими непрерывный сигнал (модулированный или немодулированный). Это достигается за счет излучения серии импульсов, прием которых осуществляется в стробоскопическом приемнике с переменным коэффициентом усиления [1,2]. Также импульсный локатор позволяет непосредственно оценивать глубину залегания обнаруженного подповерхностного объекта по времени задержки принятого сигнала, если известна, хотя бы приблизительно, скорость распространения электромагнитной волны в зондируемой среде. К недостаткам этого типа локаторов, имеющих широкий непрерывный спектр излучаемого сигнала, можно отнести потенциальную угрозу интерференции с другими источниками электромагнитных сигналов, например, спут- никовых навигационных систем и мобильной связи. Несколько лет назад в США это привело к конфликту с Федеральной комиссией по связи, которая попыталась запретить использование подповерхностных локаторов $[9,10]$.

Кроме импульсных радиолокаторов, в подповерхностной радиолокации также применяются локаторы, использующие непрерывное излучение [1]. К ним относятся локаторы с частотной модуляцией, многочастотные локаторы, излучающие когерентную сетку частот, и голографические радиолокаторы, которым посвящена настоящая работа.

Исследования в области голографической подповерхностной радиолокации были начаты только в 80-х годах в работах G. Junkin и A.P. Anderson $[11,12]$. Но, как показали их исследования, для традиционных в то время приложений, например для обследования грунтов, обладающих высоким удельным поглощением электромагнитных волн, эффективность голографических радиолокаторов оказалась недостаточно высокой из-за малой глубины зондирования [12] по сравнению с импульсными радиолокаторами, для которых глубины зондирования могли достигать $10 \mathrm{~m}$ и более. Однако в 90-х годах были разработаны голографические подповерхностные радиолокаторы серии „РАСКАН“ и был предложен целый рад приложений, которые не требовали больших глубин зондирования, но предъявляли достаточно высокие требования к пространственному разрешению с тем, чтобы получить возможность по отраженному от подповерхностного объекта сигналу определять его форму и назначение $[5,13]$. Учитывая, что локаторы „РАСКАН“ начали производиться серийно, это дало толчок дальнейшим исследования в этом направлении $[6,14]$. 


\section{1. Голографические подповерхностные радиолокаторы}

Конструкция подповерхностных локаторов базируется на классических принципах радиолокации. Сигнал, излучаемый в среду передающей антенной радиолокатора, отражается от локальной неоднородности, если ее диэлектрическая проницаемость отличается от диэлектрической проницаемости среды. Отраженный сигнал регистрируется приемной антенной радиолокатора и затем усиливается в приемнике.

Но, в отличие от импульсных локаторов, измерения в одной точке монохроматическим голографическим радиолокатором не несут никакой специфической информации, поэтому осуществляют регистрацию отра-

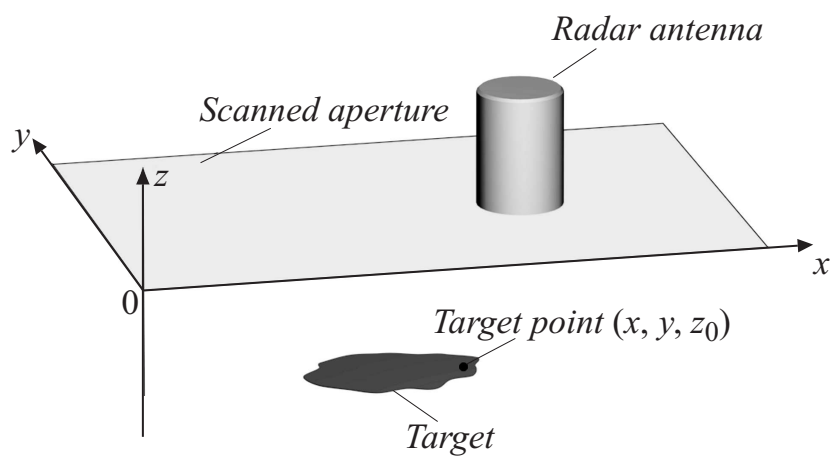

Рис. 1. Схема регистрации радиоголограммы подповерхностным локатором, где $(x, y, z)$ - декартова система координат, a $z_{0}$ - глубина залегания объекта относительно плоскости зондирования. женного сигнала на некотором участке поверхности с тем, чтобы получить радиоголограмму (рис. 1).

При этом радиосигнал, излучаемый передающей антенной, отражается от подповерхностного объекта (предметная волна) и при регистрации в приемнике смешивается с сигналом, полученным от генератора (опорная волна). В качестве опорной волны может также использоваться сигнал прямого прохождения от передающей антенны в приемную, эта схема используется при регистрации амплитудных голограмм. Особенностью исследуемой схемы голографического подповерхностного локатора является то обстоятельство, что и передающая и приемная вибраторные антенны помещены в общий корпус, представляющий собой цилиндрический волновод с открытым концом. Диаметр волновода, расположение антенн и другие параметры выбраны таким образом, чтобы обеспечить максимальную чувствительность радиолокатора [15].

Принцип получения радиоголограмм с использованием голографического подповерхностного радиолокатора можно пояснить аналогией с оптикой. Пусть плоская монохроматическая волна с постоянной фазой (опорная волна) падает на некоторый объект и рассеивается им. В результате суммирования опорной и рассеянных объектом волн на плоском экране, расположенном на некотором расстоянии за объектом, формируется интерференционная картина (рис. 2,a). Если опорная волна падает на экран по нормали, то в результате интерференции образуется голограмма, представляющая собой зонную пластину Френеля, состоящую из концентрических колец, которая имеет свойство фокусирующей линзы. После освещения опорной волной зарегистрированной

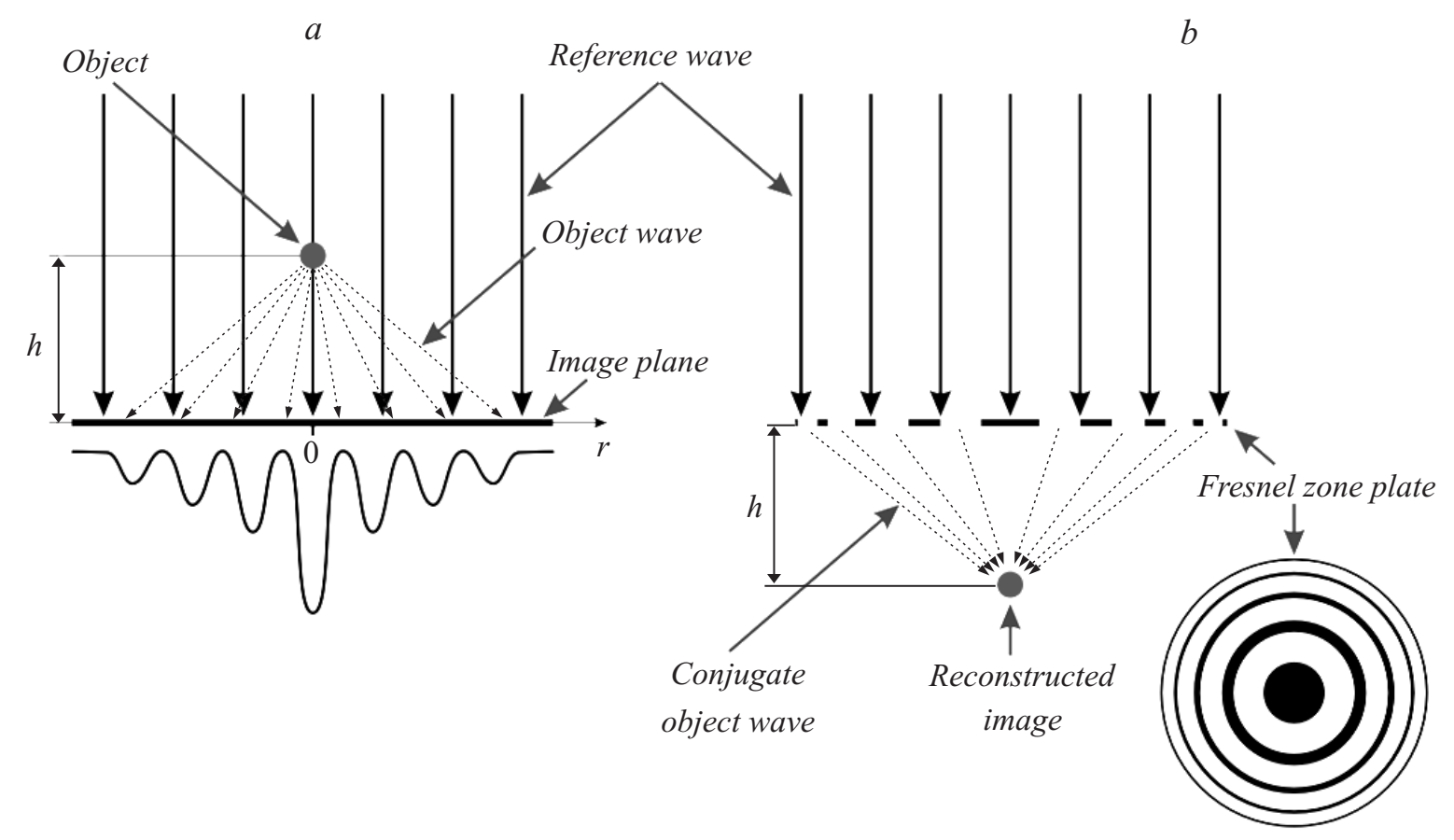

Рис. 2. Регистрация простейшей оптической голограммы $(a)$ и ее восстановление $(b)$. 


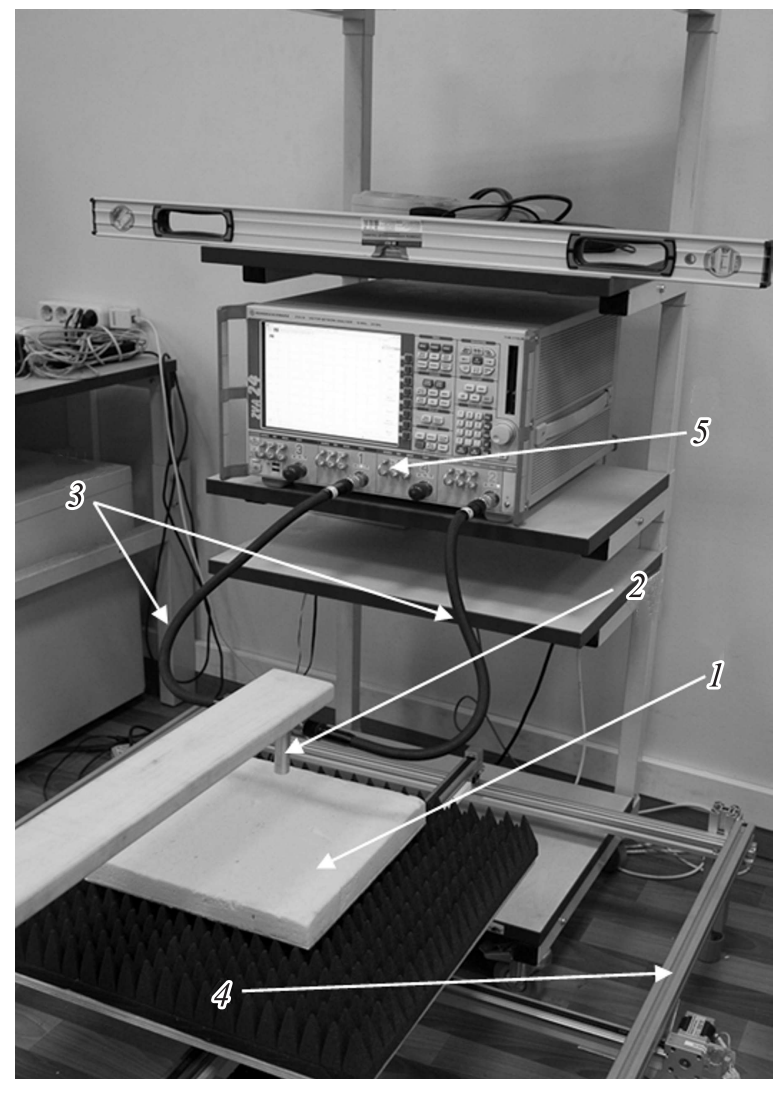

Рис. 3. Устройство экспериментальной установки: 1 - исследуемый образец, 2 - антенна, 3 - кабели, 4 - электромеханический сканер, 5 - векторный анализатор цепей ZVA 24.

голограммы формируется действительное изображение объекта (рис. 2,b). Аналогичное явление имеет место, когда СВЧ голограмма точечной мишени в однородной среде регистрируется подповерхностным голографическим радиолокатором [14]. Тем не менее существуют существенные отличия между оптической голографией и голографией в подповерхностной радиолокации.

Во-первых, если оптические голограммы регистрируются в оптически прозрачных средах с незначительным или отсутствующим затуханием, то, как правило, в подповерхностной радиолокации зондируемые среды имеют высокий коэффициент затухания - иногда $25 \mathrm{~dB} / \mathrm{m}$ и более в диапазоне длин волн, используемых для их просвечивания $[1,2]$. Это обстоятельство имеет существенное значение. Так, при большом коэффициенте затухания подповерхностный объект будет наблюдаться только по оси главного лепестка диаграммы направленности антенны (ДНА). При отклонении от оси главного лепестка для типов антенн, используемых в подповерхностной радиолокации, происходит достаточно резкое уменьшение коэффициента усиления антенны, и в средах с высоким затуханием объект не наблюдается под косыми углами, т.е. интерференционная картина отсутствует, и голограмма не регистрируется [16]. Вместо радиоголограммы в этом случае, например на бетоне, регистрируется практически теневая картина, напоминающая рентгеновское изображение [7,14].

Во-вторых, существенным отличием радиоголограмм от оптических является отношение размера системы $d$, в которой происходит регистрация голограммы, к длине волны $\lambda$. В оптике этот параметр может составлять $d / \lambda \cong 10^{6}$. Этот же параметр определяет и количество наблюдаемых интерференционных линий на наблюдаемой голограмме. На радиоголограммах, регистрируемых в подповерхностной радиолокации, $d / \lambda$ составляет только несколько единиц, и по ним можно достаточно точно определить форму регистрируемого объекта даже не прибегая к процедуре восстановления В средах с высоким поглощением, как упоминалось ранее, изображение напоминает теневое, и интерференционные линии отсутствуют.

Третьим отличием оптических и радиоголограмм является возможность регистрации в радиодиапазоне комплексных голограмм в отличие от амплитудных оптических. Это обстоятельство позволяет избавиться от мнимого изображения при восстановлении радиоголограмм и повысить их качество.

\section{2. Экспериментальная установка}

Для проведения экспериментов была создана лабораторная установка в составе векторного анализатора цепей ZVA 24 (ВАЦ), предназначенного для генерации и регистрации сигналов, антенного устройства (АУ) и электромеханического сканера (рис. 3).

Электромеханический сканер обеспечивал программное перемещение исследуемых образцов относительно неподвижного антенного устройства, представляющего собой цилиндрический волновод с открытым концом. Антенное устройство было подключено кабелями к ВАЦ. Такая схема эксперимента обеспечивает отсутствие возможного влияния переменного изгиба кабелей на регистрируемые сигналы, которое возникало бы в случае перемещения АУ.

Рабочий частотный диапазон ВАЦ дает возможность проводить эксперименты на частотах от $10 \mathrm{MHz}$ до $24 \mathrm{GHz}$, а устройство экспериментальной установки позволяет регулировать высоту расположения антенны относительно поверхности исследуемого образца. В результате возможно исследование влияния этого параметра на регистрируемые голограммы и выполнение обследования различных по толщине образцов, включая образцы с неровной поверхностью.

ВАЦ позволяет выполнять измерения не только амплитуды, но и фазы сигнала, что дает возможность регистрировать, в отличие от оптики, комплексные голограммы. Далее будет показано, что регистрация комплексных голограмм имеет существенные преимущества перед амплитудными при их восстановлении.

Величинами, измеряемыми ВАЦ в приведенных экспериментах, являлись $S$-параметры, представляющие со- 
бой коэффициенты матрицы рассеяния многополюсника

$$
\left[\begin{array}{c}
V_{1}^{-} \\
V_{2}^{\prime} \\
\vdots \\
V_{n}^{-}
\end{array}\right]=\left[\begin{array}{cccc}
S_{1} & S_{12} & \ldots & S_{1 N} \\
S_{21} & & & \vdots \\
S_{N 1} & \ldots & & S_{N M}
\end{array}\right]\left[\begin{array}{c}
V_{1}^{+} \\
V_{2}^{+} \\
\vdots \\
V_{n}^{+}
\end{array}\right],
$$

которые связывают напряжения падающей $V_{n}^{+}$и отраженной $V_{n}^{-}$волн [17]. В экспериментах измерялись значения параметров $S_{11}$ или $S_{12}$ для антенны с одним и двумя портами соответственно.

\section{3. Алгоритм восстановления радиоголограмм}

Зарегистрированные в процессе сканирования комплексные радиоголограммы нуждаются в дальнейшей обработке с помощью алгоритмов восстановления. Для восстановления использовался алгоритм, который первоначально был предложен для обработки акустических голограмм и усовершенствованный в $[18,19]$. Достоинством этого алгоритма является то обстоятельство, что в нем не используется приближение дальней зоны. В нем поперечное разрешение $\delta_{x}$ определяется только дифракционными соотношениями, и для среды без потерь выражается как

$$
\delta_{x} \approx \frac{\lambda}{2} \frac{z_{0}}{d}
$$

где $\lambda$ - длина волны излучения, $d-$ эффективный размер синтезированной апертуры с учетом диаграммы направленности. Параметр $d$ может быть существенно меньше размеров области сканирования, так как он определяется углами, под которыми отраженный от объекта сигнал регистрируется антенной.

Опишем алгоритм восстановления более подробно. Пусть плоский объект, параллельный плоскости сканирования, располагается на расстоянии $z_{0}$ от нее (рис. 1). Комплексный сигнал $E(x, y)$, зарегистрированный приемником антенны в каждой точке плоскости сканирования, можно представить как интеграл от произведения сигналов, отраженных от каждой точки объекта, и множителя, описывающего задержку отраженной волны по сравнению с опорной

$$
E(x, y)=\iint r\left(x^{\prime}, y^{\prime}\right) e^{-i 2 k \sqrt{\left(x-x^{\prime}\right)^{2}+\left(y-y^{\prime}\right)^{2}+z_{0}^{2}}} d x^{\prime} d y^{\prime},
$$

где $r\left(x^{\prime}, y^{\prime}\right)$ - функция, описывающая отражательную способность поверхности объекта (отношение комплексных амплитуд отраженной и падающей волн), $k=2 \pi f / v-$ волновое число, $v-$ скорость распространения электромагнитной волны в обследуемой среде, $f$ - частота зондирующего сигнала, двойка в показателе экспоненты возникает из-за двойного хода волны: от антенны до объекта и обратно. Скорость $v$ связана с параметрами среды соотношением

$$
v=\frac{c}{\sqrt{|\varepsilon|}},
$$

где $c$ - скорость распространения электромагнитной волны в вакууме, $\varepsilon-$ диэлектрическая постоянная среды, которая в общем случае является комплексной величиной.

В (2) не учитывается влияние ДНА и затухание сигнала в среде, влияющие на амплитуду зарегистрированного сигнала, так как по сравнению с фазой эти амплитудные зависимости играют меньшую роль при восстановлении голограмм [18]. Экспоненциальное выражение под интегралом в (2) представляет собой сферическую волну, исходящую из точки $(x, y)$, которая может быть представлена суперпозицией плоских волн [20]

$$
\begin{aligned}
& e^{-i 2 k \sqrt{\left(x-x^{\prime}\right)^{2}+\left(y-y^{\prime}\right)^{2}+z_{0}^{2}}} \\
& =\iint e^{i\left(k_{x}\left(x-x^{\prime}\right)+k_{y}\left(y-y^{\prime}\right)+k_{z} z_{0}\right)} d k_{x} d k_{y},
\end{aligned}
$$

где $k_{x}, k_{y}$ и $k_{z}$ - проекции волнового числа на соответствующие оси координат.

Подставляя (4) в (2), получим

$$
\begin{aligned}
E(x, y)= & \iint \underline{\iint r\left(x^{\prime}, y^{\prime}\right) e^{-i\left(k_{x} x^{\prime}+k_{y} y^{\prime}\right)} d k_{x^{\prime}} d k_{y^{\prime}}} \\
& \times e^{i\left(k_{x} x+k_{y} y+k_{z} z_{0}\right)} d k_{x} d k_{y} .
\end{aligned}
$$

Легко увидеть, что подчеркнутое выражение в соотношении (5) является двумерным преобразованием Фурье от функции $r(x, y)$

$$
F\left(k_{x}, k_{y}\right)=\iint r(x, y) e^{-i\left(k_{x} x+k_{y} y\right)} d x d y=\Phi_{2 \mathrm{D}}\{r(x, y)\} .
$$

Переписав (5) в виде

$$
\begin{aligned}
E(x, y) & =\iint F\left(k_{x}, k_{y}\right) e^{i k_{z} z_{0}} e^{i\left(k_{x} x+k_{y} y\right)} d k_{x} d k_{y} \\
& =\Phi_{2 \mathrm{D}}^{-1}\left\{F\left(k_{x}, k_{y}\right)\right\} e^{i k_{z} z_{0}}
\end{aligned}
$$

и используя свойства преобразования Фурье

$$
\begin{gathered}
F\left(k_{x}, k_{y}\right)=\Phi_{2 \mathrm{D}}\{E(x, y)\} e^{-i k_{z} z_{0}}, \\
r(x, y)=\Phi_{2 \mathrm{D}}^{-1}\left\{F\left(k_{z}, k_{y}\right)\right\},
\end{gathered}
$$

получим выражение для функции отражения объекта

$$
r(x, y)=\Phi_{2 \mathrm{D}}^{-1}\left\{\Phi_{2 \mathrm{D}}\{E(x, y)\} e^{-i k_{z} z_{0}}\right\} .
$$

Учитывая соотношение между волновым вектором и его проекциями

$$
k_{x}^{2}+k_{y}^{2}=k_{z}^{2}=(2 k)^{2} ; \quad k_{z}=\sqrt{4 k^{2}-k_{x}^{2}-k_{y}^{2}},
$$


получаем окончательное выражение, используемое для восстановления голограмм:

$$
r(x, y)=\Phi_{2 \mathrm{D}}^{-1}\left[\Phi_{2 \mathrm{D}}\{E(x, y)\} e^{i \sqrt{4 k^{2}-k_{x}^{2}-k_{y}^{2}} z_{0}}\right] .
$$

Как правило, в подповерхностной радиолокации глубина залегания объекта неизвестна. В этом случае мож-

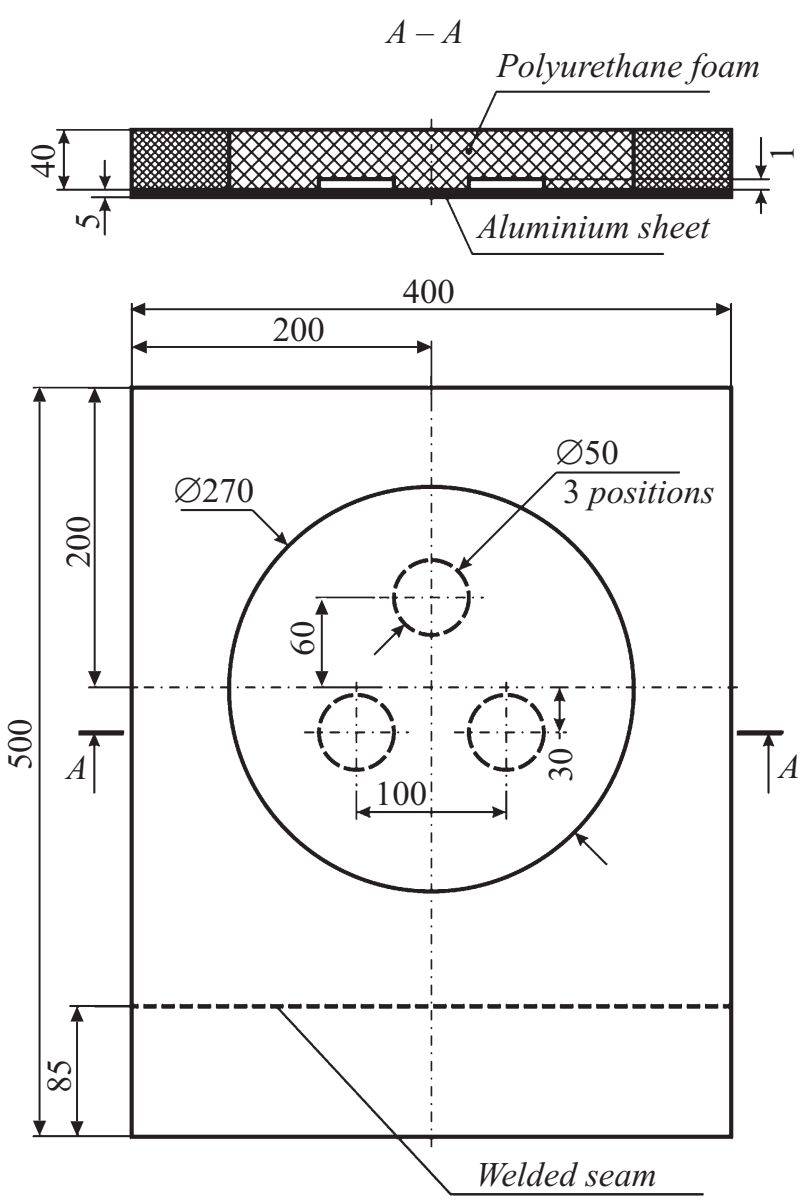

Рис. 4. Отечественный образец покрытия из вспененного полиуретана. но провести послойное восстановление голограммы с некоторым шагом по глубине, и затем выбрать глубину, где подповерхностный объект имеет наиболее четкие очертания. В некоторых сложных случаях, например для неоднородных сред с высоким уровнем затухания сигнала, этот метод может дать наилучшую фокусировку на артефакте. Впрочем, подобного рода затруднения характерны для всей подповерхностной радиолокации, так как задача определения положения подповерхностного объекта по измерениям, выполненным на ограничивающей полупространство поверхности, и к тому же с неизвестной диэлектрической проницаемостью зондируемой среды $\varepsilon$, является обратной и некорректной по своей сути.

\section{4. Экспериментальные результаты}

Ранее уже отмечалось, что регистрация и восстановление радиоголограмм возможны только для сред с относительно низким уровнем поглощения электромагнитного излучения в используемом диапазоне длин волн. Большинство сред, исследуемых в подповерхностной радиолокации, не удовлетворяют этому требованию. Однако встречаются и исключения из этого правила композиционные материалы на основе стекло- и кварцевого волокна $[21,22]$, а также вспененного полиуретана $[8,23]$. Особый интерес к исследованию покрытий из полиуретановой пены возник после катастрофы Space Shuttle Columbia в 2003 г. Этот материал, обладающий уникально низкой теплопроводностью и низким удельным весом [23,24], использовался для теплоизоляции внешнего топливного бака с криогенным топливом. Производственные дефекты, образовавшиеся в процессе нанесения покрытия, и послужили причиной отрыва куска покрытия и последующей катастрофы спускаемого аппарата [25].

Учитывая потребности аэрокосмической промышленности, был выполнен ряд исследований на экспериментальной установке, изображенной на рис. 3, по $a$

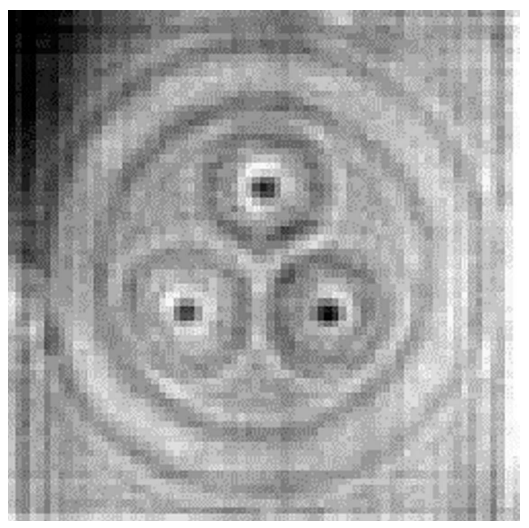

$b$

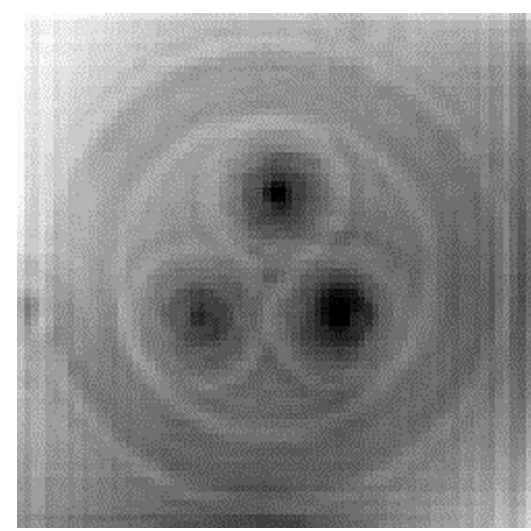

$c$

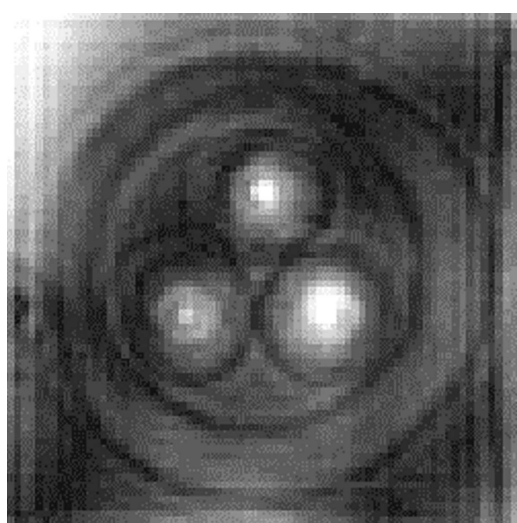

Рис. 5. Результат сканирования на частоте $20.3 \mathrm{GHz}$ образца теплоизоляции, изображенного на рис. $4: a-Q$-квадратура, $b-$ $I$-квадратура, $c$ - амплитудная голограмма. 
$a$

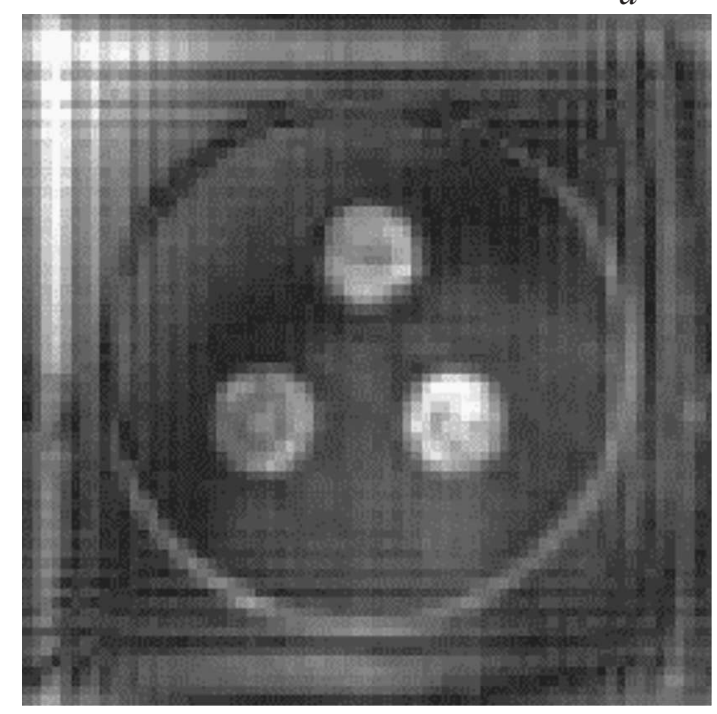

$b$

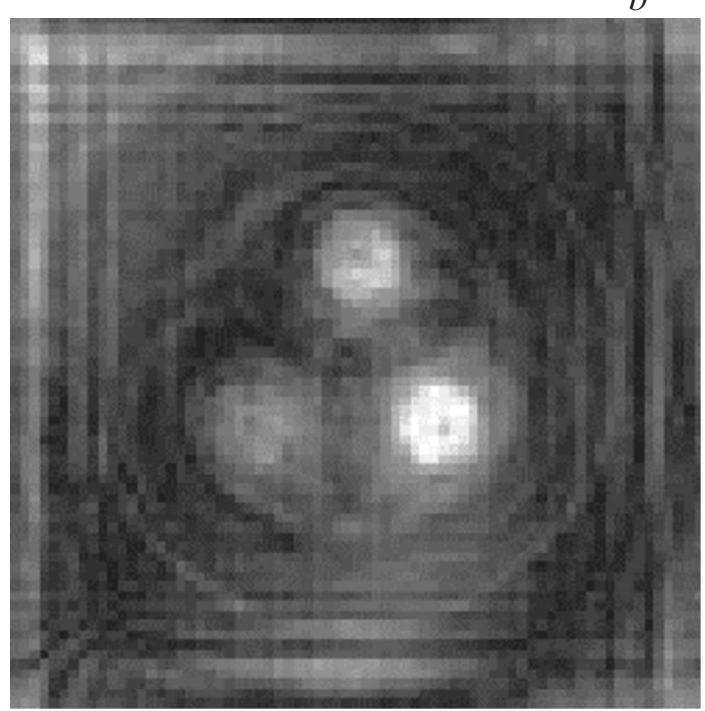

Рис. 6. Результат восстановления комплексных и амплитудных голограмм, приведенных на рис. 5: $a$ - восстановленная комплексная голограмма, $b$ - восстановленная амплитудная голограмма.

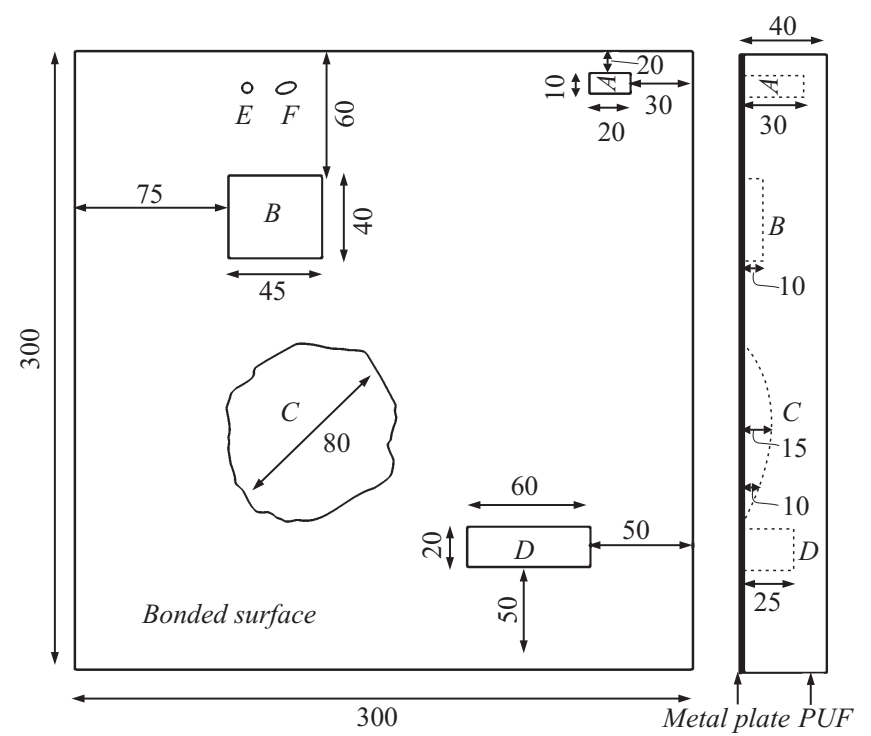

Рис. 7. Чертеж образца с дефектами, предоставленного индийским Центром космических исследований.

неразрушающему контролю диэлектрических покрытий криогенных баков перспективных ракет-носителей. В экспериментах использовались два образца покрытий, предоставленных российскими и индийскими исследовательскими центрами космической промышленности. На рис. 4 представлен чертеж отечественного образца.

Образец с искусственно произведенными дефектами представляет собой пакет теплоизоляции, полученный наклеиванием $40 \mathrm{~mm}$ слоя пенополиуретана на лист алюминиево-магниевого сплава АМг6 толщиной $5 \mathrm{~mm}$. Между слоем теплоизоляции и металлическим листом был нанесен слой грунтовки и клея общей толщи- ной $0.2 \mathrm{~mm}$. Внешняя поверхность слоя теплоизоляции подвергалась механической обработке с тем, чтобы получить ровную поверхность.

В полиуретановом слое был вырезан цилиндр диаметром $270 \mathrm{~mm}$. На внутренней поверхности цилиндра были сделаны выборки диаметром $50 \mathrm{~mm}$ и высотой $1 \mathrm{~mm}$. Затем данный цилиндр был наклеен обратно на металлическую подложку. В местах выборок на металлической поверхности отсутствовал клеевой подслой, а грунтовка и клей были нанесены на внутреннюю поверхность выборок. Полученный образец имитирует отслоение слоя теплоизоляции от поверхности бака криогенного топлива. Общая площадь образца с нанесенным слоем теплоизоляции составляет $400 \times 500 \mathrm{~mm}$.

Данный образец был помещен на стенд (рис. 3) и просканирован. Область сканирования была меньше площади образца и составляла $350 \times 350 \mathrm{~mm}$, но она покрывала всю площадь дефектов. Шаг сканирования составлял $5 \mathrm{~mm}$, а расстояние от антенны до поверхности образца было $30 \mathrm{~mm}$. Частота, на которой регистрировались радиоголограммы, составляла $20.3 \mathrm{GHz}$. Результат регистрации комплексной и амплитудной голограмм приведен на рис. 5 .

Далее зарегистрированные радиоголограммы были обработаны с помощью алгоритма восстановления, описанного в разд. 3 (1)-(12). Хотя данный алгоритм описывает процедуру восстановления более общего случая комплексных голограмм, представляло интерес сравнить их качество с восстановленными амплитудными радиоголограммами. Восстановление амплитудных радиоголограмм рассматривалось также в [26].

При восстановлении голограмм глубина фокусировки $z_{0}$ принималась равной $70 \mathrm{~mm}$, т. е. соответствовала расстоянию от среза антенны до металлической под- 
$a$

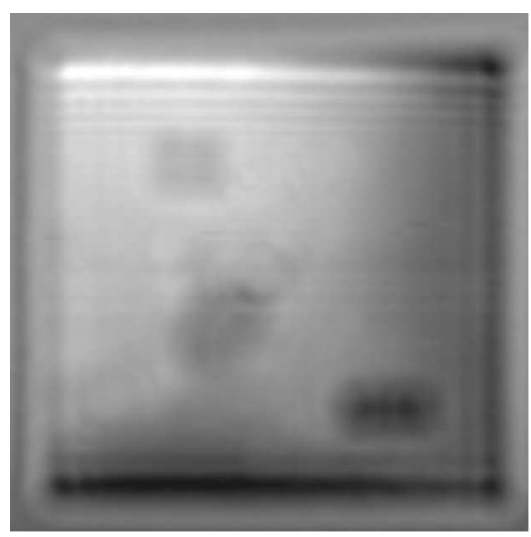

$b$

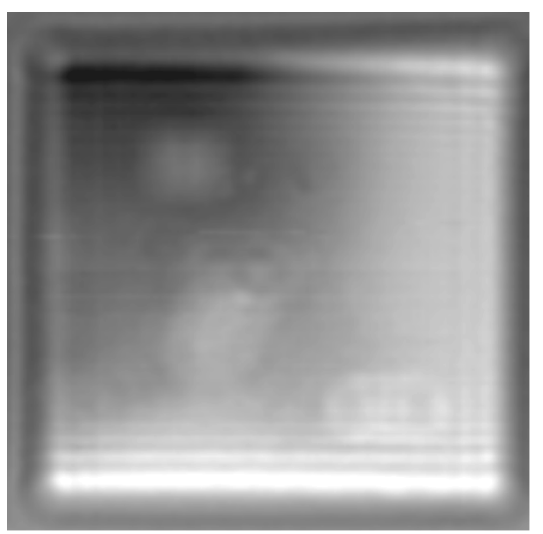

$c$

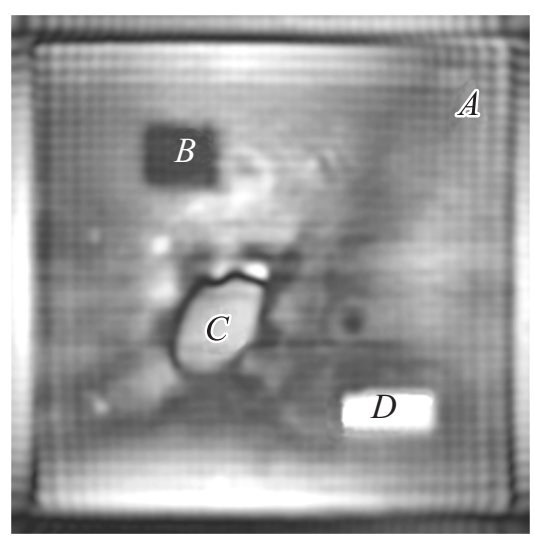

Рис. 8. Результат сканирования образца теплоизоляции, изображенного на рис. 7: $a-Q$-квадратура, $b-I$-квадратура, $c-$ восстановленная голограмма.

ложки, на которой располагались дефекты. Согласно данным [23], диэлектрическая проницаемость вспененного полиуретана, используемого на многоразовых носителях Space Shuttle, равна $\varepsilon=1.05-i 0.003$. Учитывая, что требования к теплоизоляционным материалам приблизительно одинаковые, будем считать, что во всех исследуемых в работе образцах $\varepsilon$ мало отличалась от приведенного значения. Более того, так как комплексная составляющая $\varepsilon$, определяющая электропроводность материала, очень мала, а действительная часть мало отличается от аналогичного показателя для воздуха, будем пренебрегать в алгоритмах восстановления голограмм этими отличиями и примем, что $\varepsilon=1$. Результат восстановления голограмм, приведенных на рис. 5, представлен на рис. 6.

Сравнение комплексных и амплитудных голограмм показывает, что качество изображений, полученных при восстановлении первых, существенно лучше качество восстановления вторых. Это связано с тем, что при восстановлении амплитудных голограмм происходит наложение действительного и мнимого изображений. Этот эффект при восстановлении комплексных голограмм отсутствует. На краях приведенных изображений наблюдаются полосы, наличие которых объясняется отражением электомагнитной волны от краев металлической подложки.

Еще один образец теплоизоляционного покрытия размером $300 \times 300 \mathrm{~mm}$ и толщиной $40 \mathrm{~mm}$ был предоставлен индийским Центром космических исследований в ш. Керала (рис. 7).

Оба исследуемых образца внешне были похожи между собой. Однако дефекты в индийском образце были выполнены несколько иначе. В слое пенополиуретана со стороны, обращенной к металлической подложке, были выбраны углубления различной формы и размеров. Затем слой покрытия был приклеен к подложке. Результат сканирования данного образца и зарегистрированная комплексная голограмма на частоте $22.5 \mathrm{GHz}$ приведены на рис. $8, a, b$. На рис. $8, c$ приведена восстановленная голограмма.

На восстановленном изображении были обнаружены все крупные неоднородности: $A, B, C$ и $D$. Мелкие неоднородности $E$ и $F$, размеры которых на чертеже рис. 7 не указаны, не просматриваются. Вместе с тем на изображении рис. 8,c проявились неоднородности, природа которых не определена. Возможно, они связаны с неровностями внутренней поверхности слоя пенополиуретана или клеевого слоя.

\section{Заключение}

Результаты экспериментов показывают, что предложенный метод неразрушающего контроля теплоизоляционных пенополиуретановых покрытий изделий ракетнокосмической техники с помощью голографического подповерхностного радиолокатора гигагерцового диапазона может найти применение наряду с другими методами диагностики (например, с использованием ультразвука или термографии). Для этой цели необходимо разработать специализированные датчики, оснащенные средствами сканирования крупногабаритных деталей, таких как ракетные баки, предназначенные для хранения криогенного топлива. Для достижения необходимой чувствительности к дефектам и неоднородностям в теплоизоляционных покрытиях рабочие частоты устройства должны находиться в диапазоне от 20 до $24 \mathrm{GHz}$.

Работа выполнена при поддержке грантов Российского научного фонда № 15-19-00126 (в части разработки экспериментальной установки) и № 15-19-30012 (в части разработки алгоритмов обработки зарегистрированных радиоголограмм).

Авторы выражают признательность НПО „Техномаш“ и Vikram Sarabhai Space Center, Индия, за предоставленные образцы теплоизоляции для исследований. 


\section{Список литературы}

[1] Daniels D.J. Ground Penetrating Radar. $2^{\text {nd }}$ ed. London: IEE, 2004. 734 p.

[2] Финкельштейн М.И., Карпухин В.И., Кутев А.В., Метелкин В.Н. Подповерхностная радиолокация / Под ред. М.И. Финкельштейна. М.: Радио и связь, 1994. 216 с.

[3] Финкельштейн М.И., Кутев В.А., Золотарев В.П. Применение радиолокационного подповерхностного зондирования в инженерной геологии / Под ред. М.И. Финкельштейна. М.: Недра, 1986. 128 с.

[4] Nuzzo L., Alli G., Guidi R., Cortesi N., Sarri A., Manacorda G. // Proc. $15^{\text {th }}$ Int. Conf. on Ground Penetrating Radar, 2014. P. 969-974.

[5] Ivashov S.I., Makarenkov V.I., Razevig V.V., Sablin V.N., Sheyko A.P., Vasiliev I.A. // Proc. $8^{\text {th }}$ International Conference on Ground Penetrating Radar, 2000. P. 36-39.

[6] Song X.J., Su Y., Huang C.L., Lu M., Zhu S.P. // Proc. of the $16^{\text {th }}$ Int. Conf. on Ground Penetrating Radar, 2016. P. 1-4.

[7] Razevig V.V., Ivashov S.I., Sheyko A.P., Vasilyev I.A., Zhuravlev A.V. // Proc. Prog. Electromagn. Res. Lett. 2008. Vol. 1. P. 173-179.

[8] Ivashov S., Razevig V., Vasiliev I., Bechtel T., Capineri L. // NDT E Int. J. 2015. Vol. 69. P. 48-54.

[9] Ultra-Wideband Transmission Systems. ET Docket N 98-153. First Report and Order, FCC 02-48 (released April 22, 2002).

[10] Johnson D.J. GPR - The Impact of New FCC Regulations, GSSI July 25, 2002. 4 p.

[11] Junkin G., Anderson A. P. // Proc. of the $16^{\text {th }}$ European Microwave Conference. IEEE Conference Publications. 1986. P. 720-725.

[12] Junkin G. Anderson A.P. // IEE Proc. F. Vol. 135. N 4. 1988. P. 321-329.

[13] Vasiliev I.A., Ivashov S.I., Makarenkov V.I., Sablin V.N., Sheyko A.P. // IEEE Aero. El. Sys. Mag. 1999. Vol. 14. N 5. P. 25-28.

[14] Ivashov S.I., Razevig V.V., Vasiliev I.A., Zhuravlev A.V., Bechtel T.D., Capineri L. // IEEE J. Sel. Top. Appl. 2011. Vol. 4. N 4. P. 763-778.

[15] Разевиг В.В., Васильев И.А., Ивашов А.И., Ивашов С.И., Макаренков В.И. Способ получения радиоголограмм подповерхностных объектов. Патент РФ. № 2482518. 20.05.2013.

[16] Razevig V.V., Ivashov S.I., Vasiliev I.A., Zhuravlev A.V., Bechtel T., Capineri L. // Proc. of the XIII International Conference on Ground Penetrating Radar. Lecce, Italy, 2010. P. 657-662.

[17] Pozar D.M. Microwave Engineering. $4^{\text {th }}$ ed. John Wiley \& Sons, Inc., 2012. 756 p.

[18] Sheen D.M., McMakin D.L., Hall T.E. // IEEE T. Microw. Theory. 2001. Vol. 49. N 9. P. 1581-1592.

[19] Разевиг В.В., Бугаев А.С., Ивашов С.И., Васильев И.А., Журавлев А.В. // Успехи современной радиоэлектроники. 2010. № 9. C. 51-58.

[20] Бреховских Л.М. Волны в слоистых средах. М.: Наука, 1973. $343 \mathrm{c}$.
[21] Ivashov S.I., Razevig V.V., Bechtel T.D., Vasiliev I.A., Capineri L., Zhuravlev A.V. // Proc. IEEE Int. Conf. on Microwaves, Communications, Antennas and Electronic Systems (COMCAS 2015). Tel-Aviv, Israel, 2015. P. 1-5.

[22] Lu T., Snapp C., Chao T.-H., Thakoor A., Bechtel T., Ivashov S., Vasiliev I. // Proc. SPIE. 2007. Vol. 6555. P. 65550S1-8.

[23] Kharkovsky S., Zoughi R. // IEEE Instrum Meas Mag. 2007. P. 26-38.

[24] Dombrow B.A. Polyurethanes. NY: Reinhold Publishing Corporation, 1957. $185 \mathrm{p}$.

[25] Morring Jr. F. // Aviat. Week Space Tec. 2003. P. 31.

[26] Суханов Д.Я., Завьялова К.В. // ЖТФ. 2012. Т. 82. Вып. 6. C. $85-89$. 